\title{
An unusual white dwarf star may be a surviving remnant of a subluminous Type Ia supernova
}

\author{
S. Vennes ${ }^{1 *}$, P. Nemeth ${ }^{2,3}$, A. Kawka ${ }^{1}$, J.R. Thorstensen ${ }^{4}$, \\ V. Khalack ${ }^{5}$, L. Ferrario ${ }^{6}$ \& E.H. Alper ${ }^{4}$ \\ ${ }^{1}$ Astronomický ústav, Akademie věd České republiky, \\ Fričova 298, CZ-251 65 Ondřejov, Czech Republic. \\ 2 Dr. Karl Remeis-Sternwarte, Astronomical Institute, \\ University Erlangen-Nürnberg, Sternwartstr. 7, 96049 Bamberg, Germany. \\ ${ }^{3}$ Astroserver.org, 8533 Malomsok, Hungary \\ ${ }^{4}$ Department of Physics and Astronomy, 6127 Wilder Laboratory, Dartmouth College, \\ Hanover, NH 03755-3528, USA. \\ ${ }^{5}$ Département de physique et d'astronomie, Université de Moncton, \\ Moncton, New Brunswick E1A 3E9, Canada. \\ ${ }^{6}$ Mathematical Sciences Institute, The Australian National University, \\ Canberra, ACT 0200, Australia. \\ * Corresponding author. E-mail: vennes@ asu.cas.cz. \\ received 23 January 2017; accepted 18 July 2017
}

Subluminous Type Ia supernovae, such as the Type Iax class prototype SN 2002cx, are described by a variety of models such as the failed detonation and partial deflagration of an accreting carbon-oxygen white dwarf star, or the explosion of an accreting, hybrid carbon-oxygen-neon core. These models predict that bound remnants survive such events with, according to some simulations, a high kick velocity. We report the discovery of a high proper motion, low-mass 


\section{white dwarf (LP 40-365) that travels at a velocity greater than the Galactic escape velocity and whose peculiar atmosphere is dominated by intermediate- mass elements. Strong evidence indicates that this partially burnt remnant was ejected following a subluminous Type Ia supernova event. This supports the viability of single-degenerate supernova progenitors.}

Type Ia supernova (SN Ia) explosions are powered by the detonation of a Chandrasekharmass white dwarf with a degenerate carbon-oxygen core (1). Models show that the explosion may be triggered by the high internal pressure caused either by matter accreted from a close donor star [the single degenerate (SD) scenario] or by the merger with another white dwarf (the double degenerate scenario) (2). Although Type Ia supernovae are used to calibrate the cosmological distance scale (1) and constrain cosmological models (3,4), our knowledge of these objects is incomplete and their progenitors have remained elusive $(5,6)$. The possibility of detecting surviving remnants from subluminous SN Ia events may help illuminate the SN Ia progenitor problem in general. Models (7-9) proposed to explain observed properties of subluminous SN Ia such as the SN Iax-class prototype SN 2002cx $(10,11)$ involve failed detonation and partial deflagration of a massive white dwarf (7-9) or the explosion of a hybrid carbon-oxygen-neon $(\mathrm{CONe})$ core $(12,13)$ with both scenarios expected, under the right circumstances, to leave a bound remnant. Direct evidence for such remnants is missing (5).

We have observed the high proper motion star LP 40-365 (14). An identification spectrum was obtained on 2015 February 21 using the Richtey-Chretien spectrograph attached to the Mayall 4-m telescope at Kitt Peak National Observatory (KPNO) (Fig. 1). The main characteristics are a blue continuum indicating a temperature of $\approx 10000 \mathrm{~K}$ (circa B9 star); the complete absence of neutral hydrogen or helium absorption lines, unlike in normal B stars; and the appearance of strong magnesium (Mg I-II) and sodium ( $\mathrm{Na}$ I) line series and weaker lines of oxygen (O I). Table 1 lists astrometric (15) and photometric (16) data for this object. We 
followed up this unusual spectrum using low- to high-dispersion spectra obtained between June 2015 and June 2016 with the William Herschel 4.2-m telescope on La Palma, the Hiltner 2.4-m telescope on Kitt Peak, and, finally, with the Gemini-North 8-m telescope on Mauna Kea (17).

We performed a spectral line analysis using an iterative procedure that adjusts a parametrized spectral synthesis to the observed line spectrum using $\chi^{2}$ minimization techniques. These calculations were performed by using a multi-parameter fitting procedure that constrains simultaneously the effective temperature $\left(T_{\text {eff }}\right)$ and surface gravity $(\log g)$ of the star and each individual element abundance in the atmosphere (17). We analyzed the high-resolution spectra obtained with the Echelle SpectroPolarimetric Device for the Observation of Stars (ESPaDOnS) fed by optical fibers attached to the Gemini-North telescope using the Gemini Remote Access to CFHT ESPaDOnS Spectrograph (GRACES) (CFHT, Canada-France-Hawaii Telescope).

The model atmospheres and synthetic spectra supporting our analysis were calculated in full non-local thermodynamic equilibrium (non-LTE) by using the computer codes TLUSTY version 204 and SYNSPEC version $49(17,18)$. The chemical composition includes elements with atomic numbers from $Z=1(\mathrm{H})$ to $30(\mathrm{Zn})$ with all relevant ionized atoms. The atmosphere is in radiative equilibrium; convective energy transport was found to be inefficient. Detailed line profiles were calculated by using line-broadening parameters dominated by electronic collisions (Stark effect). Table 1 lists best-fitting stellar parameters (with $1 \sigma$ statistical error bars) and Figure 2 shows the corresponding abundances. The high effective temperature and the surface gravity, which is intermediate between normal white dwarfs and the main sequence stars, indicate that this object is most likely a low-mass degenerate star (19). The line profiles demonstrate that the star is rotating with a projected rotation velocity $v \sin i=30.5 \mathrm{~km} \mathrm{~s}^{-1}$, where $i$ is the apparent inclination of the rotation axis and $v$ is the equatorial rotation velocity, suggesting that the parent body was spun up during binary interaction. The abundance analysis shows that the main atmospheric constituents are oxygen and neon with substantial traces of intermediate-mass elements 
such as aluminium and silicon.

We measured a large Doppler wavelength shift in the spectral line analysis. From a sample of 21 velocity measurements taken at different epochs and after correcting for Earth's motion, we measured an average radial velocity $v_{\mathrm{r}}=497.6 \pm 1.1 \mathrm{~km} \mathrm{~s}^{-1}$, without significant variations $\left(\chi_{\mathrm{r}}^{2}=1.3\right)$. Therefore the star is apparently single and moving at a velocity characteristic of hyper-velocity stars (20). Those objects are former members of binary systems that were ejected during three-body encounters with the Galactic center (GC) or ejected following the demise of a massive white dwarf companion in a SD SN Ia (21).

In addition to its large radial velocity, LP 40-365 shows a large apparent motion across the celestial sphere of $\mu=158$ milli-arc sec per year $\left(\right.$ mas $^{-1} r^{-1}$ ). With knowledge of the distance $d$, the proper motion vector may be converted into the tangential velocity vector $v_{\mathrm{T}}$, which combined with the radial velocity $v_{\mathrm{r}}$ provides a complete 3 -dimensional description of LP 40365's motion. We estimated the distance toward LP 40-365 with a photometric method (17) using as inputs the apparent luminosity of the star and an estimate of its absolute lumimosity. The absolute luminosity is calculated by using the surface temperature measurements described earlier and an estimate of the stellar radius which is model dependent. However, that distance estimate will eventually be superseded by Gaia parallax measurements (22).

The radius of a low-mass, degenerate or partly degenerate body is sensitive to finite-temperature effects $(23,24)$ that would inflate the radius of a young, extremely low-mass white dwarf such as LP 40-365. Models for carbon, oxygen, silicon, or iron cores are available (25), but unfortunately the predicted surface gravity of available models $(\log g>6.0$, where $g$ is expressed in $\mathrm{cm} \mathrm{s}^{-2}$ ) largely exceeds the measured gravity of LP 40-365 indicating that its mass should be much lower than 0.3 solar mass $\left(\mathrm{M}_{\odot}\right)$. Lower-mass models with helium interiors are available $(23)$ and indicate that a body with a mass of $\approx 0.14 \mathrm{M}_{\odot}$ and $\approx 8 \%$ of the solar radius $\left(\mathrm{R}_{\odot}\right)$ reproduces the surface gravity and effective temperature of LP 40-365, assuming a cooling age 
between 5 and 50 million years (17). Although we have concluded that the interior of LP 40365 is most likely composed of carbon, oxygen and neon, or heavier elements, helium models characterized by identical mean electronic weight $\left(\mu_{e}=2\right)$ represent a reasonable proxy. The central temperature of the adopted model, $T_{\mathrm{c}} \approx 30 \times 10^{6} \mathrm{~K}$, is lower than that of a typical inert core of normal white dwarfs (23). Adopting a radius of $0.078 \mathrm{R}_{\odot}$ we estimated an absolute magnitude in the Johnson $\mathrm{V}$ band $M_{\mathrm{V}}=8.14$ mag. Thus, the apparent $m_{\mathrm{V}}$ magnitude listed in Table 1 implies a distance of $298_{-70}^{+150} \mathrm{pc}$. The tangential velocity at a distance of $298 \mathrm{pc}$ is $v_{\mathrm{T}}=224 \mathrm{~km} \mathrm{~s}^{-1}$ for a total space velocity relative to the Sun of $546 \mathrm{~km} \mathrm{~s}^{-1}$.

To retrace the past history of this object, we converted the apparent velocity components (radial and tangential) into the Galactic velocity vector $(26)(U, V, W)=(-346,360,217) \mathrm{km} \mathrm{s}^{-1}$. This instantaneous velocity vector may be projected back in time by adopting an appropriate Galactic potential model (27). We followed the Galactic orbit of LP 40-365 from the present time $(t=0)$ back to $t=-100$ million years. The projected trajectories displayed in Fig. 3 indicate that, for an assumed starting point set at distances between 100 and $1000 \mathrm{pc}$, the object did not encounter the GC and, therefore, is not the product of a three-body dynamical interaction with the GC (28). None of the resultant trajectories, which allowed for uncertainties in the distance, are bound Galactic orbits either. The total velocity in the Galactic rest frame varies between $675 \mathrm{~km} \mathrm{~s}^{-1}$ assuming $d=100 \mathrm{pc}$ and $1016 \mathrm{~km} \mathrm{~s}^{-1}$ assuming $d=1000 \mathrm{pc}$ with a velocity of $709 \mathrm{~km} \mathrm{~s}^{-1}$ at the distance (298 pc) set by the photometric method. All exceed the Galactic escape velocity at $8.5 \mathrm{kpc}$ from the GC (20). The object must have originated along one of those projected trajectories and the trajectory that took LP 40-365 to the present-day distance of $298 \mathrm{pc}$ entered the plane $<5$ million years ago. The simulated cooling time scale for a $0.15 \mathrm{M}_{\odot}$ compact object with an effective temperature of $10^{4} \mathrm{~K}$ is only $\approx 5$ to 50 million years $(23)$.

Combining the peculiar surface composition of this compact object, the results of the trajectory analysis, and the evolutionary age estimate, it appears likely that LP 40-365 is the surviving 
remnant of a subluminous SN Ia event that took place below the Galactic plane, a few kiloparsecs away and earlier than 50 million years ago. The stellar properties and the kinematics of LP 40-365 are comparable to some simulated events (7), suggesting that this object is indeed a fragment that survived the failed detonation of an SN Iax. The mass estimate is somewhat less than accounted for in these simulations $\left(>0.3 \mathrm{M}_{\odot}\right)$. However, other models (9) successfully achieve remnants with masses as low as $0.09 \mathrm{M}_{\odot}$ but without delivering a large kick velocity. Variations in the adopted ignition geometry, such as centered versus off-center ignition, may affect the kinematical outcome for the surviving remnant. Simulations involving hybrid CONe cores (13) successfully generated low-mass remnants but these simulations did not explore postexplosion kinematics.

Intermediate-mass elements detected in the atmosphere of LP 40-365 are expected to contaminate bound remnants after a typical SN Iax event (9), but we found only minute traces of iron-group elements which normally dominate the supernova ejecta. The paucity of iron-group elements and the prevalence of lighter elements indicate that gravitational settling and chemical separation may have occurred with light elements dominating over heavier ones. It is not possible to estimate the fraction of iron material produced in the explosion that would manage to diffuse to the star's center. Diffusion time scales at the star's surface may be comparable to or longer than the age of the object (29). Conversely, the absence of carbon and prevalence of oxygen and neon at the surface of LP 40-365 would preferably match the configuration of a hybrid CONe core (13). None of the simulations take element diffusion explicitly into account, therefore a detailed comparison of predicted and observed surface compositions would not constitute a definitive test for any models.

It has been suggested that dynamical instability in a low-mass $\mathrm{x}$-ray binary orbiting a distant main-sequence star could result in the high-velocity ejection of the donor star (30,31). Apart from an unspecified surface composition, the predicted high-velocity star could resemble LP 40- 
365. However, only $\sim 10^{-8}$ such events are expected per year in the Milky Way (31) compared to a rate of $\sim 10^{-3}$ for SN Iax events (32); therefore this scenario is less probable.

The actual donor star that must also have been ejected (21) along with LP 40-365 should be detectable as well. For example, the high-velocity, helium-rich subdwarf star US 708 (33) is a representative of the class of donor stars that emerged from a SD SN Ia event and a similar object would have been ejected along with LP 40-365 after the proposed underluminous SN Ia event. The possible detection of a bound remnant in the aftermath of the SN Iax event SN 2008ha has been reported although it may be a chance alignment (32). The properties of that object are unknown. The tentative progenitor of SN $2012 \mathrm{Z}$ has been described as nova-like (34) suggesting the likely presence of an accreting white dwarf in a SD progenitor system akin to that of LP 40365. No bound remnant has been identified. The atmospheric properties of LP 40-365 share some similarities with those of another extreme white dwarf (35) but exhibit clear distinctions as well: Both WD 1238+674 and LP 40-365 are oxygen-rich but WD 1238+674 is more massive $\left(0.6 \mathrm{M}_{\odot}\right.$ versus $\left.0.14 \mathrm{M}_{\odot}\right)$ and its kinematical properties do not appear as extreme. The discovery of the oxygen-neon white dwarf WD 1238+674 lends support to the hybrid CONe formation model (36) and, indirectly, to the subluminous SNIa explosion models involving hybrid CONe white dwarfs (13). 


\section{References and Notes}

1. K. Nomoto, K. Iwamoto, N. Kishimoto, Type Ia supernovae: their origin and possible applications in cosmology. Science 276, 1378-1382 (1997).

2. I. Iben Jr., A. V. Tutukov, Supernovae of type I as end products of the evolution of binaries with components of moderate initial mass ( $\mathrm{M}$ not greater than about 9 solar masses). Astrophys. J. Supp. Ser. 54, 335-372 (1984).

3. A. G. Riess et al., Observational Evidence from Supernovae for an Accelerating Universe and a Cosmological Constant. Astron. J. 116, 1009-1038 (1998).

4. S. Perlmutter et al., Measurements of $\Omega$ and $\Lambda$ from 42 High-Redshift Supernovae. Astrophys. J. 517, 565-586 (1999).

5. D. Maoz, F. Mannucci, G. Nelemans, Observational Clues to the Progenitors of Type Ia Supernovae. Annu. Rev. Astron. Astrophys. 52, 107-170 (2014).

6. B. Wang, Z. Chan, Progenitors of type Ia supernovae. New Astronomy Reviews 56, 122-141 (2012).

7. G. C. Jordan IV, H. B. Perets, R. T. Fisher, D. R. van Rossum, Failed-detonation Supernovae: Subluminous Low-velocity Ia Supernovae and their Kicked Remnant White Dwarfs with Iron-rich Cores. Astrophy. J. 761, L23 (2012).

8. M. Kromer et al., 3D deflagration simulations leaving bound remnants: a model for 2002cxlike Type Ia supernovae. Mon. Not. R. Astron. Soc. 429, 2287-2297 (2013).

9. M. Fink, M. Kromer, I. R. Seitenzahl, F. Ciaraldi-Schoolmann, F. K. Röpke, S. A. Sim, R. Pakmor, A. J. Ruiter, W. Hillebrandt, Three-dimensional pure deflagration models with 
nucleosynthesis and synthetic observables for Type Ia supernovae. Mon. Not. R. Astron. Soc. 438, 1762-1783 (2014).

10. D. Branch, E. Baron, R. C. Thomas, D. Kasen, W. Li, A. V. Filippenko, Reading the Spectra of the Most Peculiar Type Ia Supernova 2002cx. Publ. Astron. Soc. Pac. 116, 903-908 (2004).

11. R. J. Foley et al., Type Iax Supernovae: A New Class of Stellar Explosion. Astrophys. J. 767, 57 (2013).

12. M. Kromer et al., Deflagrations in hybrid CONe white dwarfs: a route to explain the faint Type Iax supernova 2008ha. Mon. Not. R. Astron. Soc. 450, 3045-3053 (2015).

13. E. Bravo, P. Gil-Pons, J.L. Gutiérrez, C.L. Doherty, Explosion of white dwarfs harboring hybrid CONe cores. Astron. Astrophys. 589, A38 (2016).

14. W. J. Luyten, Proper Motion Survey with the 48-inch Schmidt telescope. XXII. Special catalogues for the zone +70 to +75 (Univ. of Minnesota Press, 1970).

15. S. Lépine, M. M. Shara, A Catalog of Northern Stars with Annual Proper Motions Larger than 0.15" (LSPM-NORTH Catalog). Astron. J. 129, 1483-1522 (2005).

16. C. Watson, A. A. Henden, A. Price, VizieR Online Data Catalog: AAVSO International Variable Star Index VSX (Watson+, 2006-2014) (2016).

17. Materials and methods are available as supplementary materials.

18. I. Hubeny, T. Lanz, Non-LTE line-blanketed model atmospheres of hot stars. 1: Hybrid complete linearization/accelerated lambda iteration method. Astrophys. J. 439, 875-904 (1995). 
19. S. Gianninas, P. Dufour, M. Kilic, W. R. Brown, P. Bergeron, J. J. Hermes, Precise Atmospheric Parameters for the Shortest-period Binary White Dwarfs: Gravitational Waves, Metals, and Pulsations. Astrophys. J. 794, 35 (2014).

20. W. R. Brown, Hypervelocity Stars. Annu. Rev. Astron. Astrophys. 53, 15-49 (2015).

21. S. Justham, C. Wolf, P. Podsiadlowski, Z. Han, Type Ia supernovae and the formation of single low-mass white dwarfs. Astron. Astrophys. 493, 1081-1091 (2009).

22. M. A. C. Perryman et al., GAIA: Composition, formation and evolution of the Galaxy. Astron. Astrophys. 369, 339-363 (2001).

23. L. G. Althaus, O. G. Benvenuto, Evolution of Helium White Dwarfs of Low and Intermediate Masses. Astrophys. J. 477, 313-334 (1997).

24. O. G. Benvenuto, L. G. Althaus, Grids of white dwarf evolutionary models with masses from $M=0.1$ to $1.2 \mathrm{M}_{\odot}$. Mon. Not. R. Astron. Soc. 303, 30-38 (1999).

25. J. A. Panei, L. G. Althaus, O. G. Benvenuto, Mass-radius relations for white dwarf stars of different internal compositions. Astron. Astrophys. 353, 970-977 (2000).

26. D. R. H. Johnson, D. R. Soderblom, Calculating galactic space velocities and their uncertainties, with an application to the Ursa Major group. Astron. J. 93, 864-867 (1987).

27. C. Allen, A. Santillan, An improved model of the galactic mass distribution for orbit computations. Revista Mexicana de Astronomia y Astrofisica 22, 255-263 (1991).

28. J. G. Hills, Hyper-velocity and tidal stars from binaries disrupted by a massive Galactic black hole. Nature 331, 687-689 (1988). 
29. C. Paquette, C. Pelletier, G. Fontaine, G. Michaud, Diffusion in white dwarfs - New results and comparative study. Astrophys. J. Supp. Ser. 61, 197-217 (1986).

30. P. C. C. Freire et al., On the nature and evolution of the unique binary pulsar J1903+0327. Mon. Not. R. Astron. Soc. 412, 2763-2780 (2011).

31. S. Portegies Zwart, E. P. J. van den Heuvel, J. van Leeuwen, G. Nelemans, The Formation of the Eccentric-orbit Millisecond Pulsar J1903+0327 and the Origin of Single Millisecond Pulsars. Astrophys. J. 734, 55 (2011).

32. R. J. Foley, C. McCully, S. W. Jha, L. Bildsten, W.-f. Fong, G. Narayan, A. Rest, M. D. Stritzinger, Possible Detection of the Stellar Donor or Remnant for the Type Iax Supernova 2008ha. Astrophys. J. 792, 29 (2014).

33. S. Geier et al., The fastest unbound star in our Galaxy ejected by a thermonuclear supernova. Science 347, 1126-1128 (2015).

34. C. McCully, S. W. Jha, R. J. Foley, L. Bildsten, W.-F. Fong, R. P. Kirshner, G. H. Marion, A. G. Riess, M. D. Stritzinger, A luminous, blue progenitor system for the type Iax supernova 2012Z. Nature 512, 54-56 (2014).

35. S. O. Kepler, D. Koester, G. Ourique, A white dwarf with an oxygen atmosphere. Science 352, 67-69 (2016).

36. C.L. Doherty, P. Gil-Pons, L. Siess, J.C. Lattanzio, H.H.B. Lau, Super- and massive AGB stars - IV. Final fates - initial-to-final mass relation. Mon. Not. R. Astron. Soc. 446, 2599-2612 (2015). 


\section{Acknowledgments}

A.K. and S.V. acknowledge support from the Grant Agency of the Czech Republic (15-15943S). A.K. was a visiting astronomer at KPNO, National Optical Astronomy Observatory. This work was also supported by the project RVO:67985815 in the Czech Republic. The research leading to these results has received funding from the European Community's Seventh Framework Programme (FP7/2013-2016) under grant agreement number 312430 [Optical Infrared Coordination Network for Astronomy (OPTICON)]. J.R.T. acknowledges support from NSF grant AST-1008217. This research made use of services at Astroserver.org under the reference number NWKZKA.

This research is based on observations obtained at KPNO, National Optical Astronomy Observatory, and at the Gemini Observatory, program GN-2016A-FT-24, using ESPaDOnS, located at the CFHT. This publication makes use of data products from the Wide-field Infrared Survey Explorer, the Two Micron All Sky Survey, and the American Association of Variable Sky Observers (AAVSO) Photometric All Sky Survey (APASS). Complete acknowledgments are offered as supplementary materials on Science Online. Observational data are available in publicly available archives, as are our calculated model atmospheres. Full details and URLs are given in the supplementary material.

\section{Supplementary materials}

Materials and Methods

Supplementary Text

Figs. S1 to S8

Tables S1 and S2

References (37-58) 
Table 1: Stellar data and parameters. The celestial coordinates are provided along the right ascension $(\mathrm{RA} \equiv \alpha)$ and declination $(\mathrm{Dec} \equiv \delta)$ and the apparent motion of the star, i.e., the proper motion $\mu$, is decomposed into $\mu_{\alpha} \cos \delta$ along the right ascension and $\mu_{\delta}$ along the declination.

\begin{tabular}{lc}
\hline \hline Parameter & Measurement \\
\hline RA $(\mathrm{J} 2000)$ & $14^{\mathrm{h}} 06^{\mathrm{m}} 35^{\mathrm{s}} .45$ \\
Dec $(\mathrm{J} 2000)$ & $+74^{\circ} 18^{\prime} 58^{\prime \prime} .0$ \\
$\mu_{\alpha} \cos \delta$ & $-56 \pm 7$ mas year $^{-1}$ \\
$\mu_{\delta}$ & $148 \pm 7$ mas year $^{-1}$ \\
$m_{\mathrm{V}}$ & $15.51 \pm 0.09 \mathrm{mag}$ \\
$T_{\text {eff }}$ & $10100_{-350}^{+250} \mathrm{~K}$ \\
$\log g /\left(\mathrm{cm} \mathrm{s}^{-2}\right)$ & $5.80_{-0.35}^{+0.20}$ \\
Mass & $0.14 \pm 0.01 \mathrm{M}_{\odot}$ \\
Radius & $0.078_{-0.020}^{+0.040} \mathrm{R}_{\odot}$ \\
$M_{\mathrm{V}}$ & $8.14_{-0.90}^{+0.60} \mathrm{mag}$ \\
$v \sin i$ & $30.5 \pm 2.0 \mathrm{~km} \mathrm{~s}^{-1}$ \\
\hline
\end{tabular}




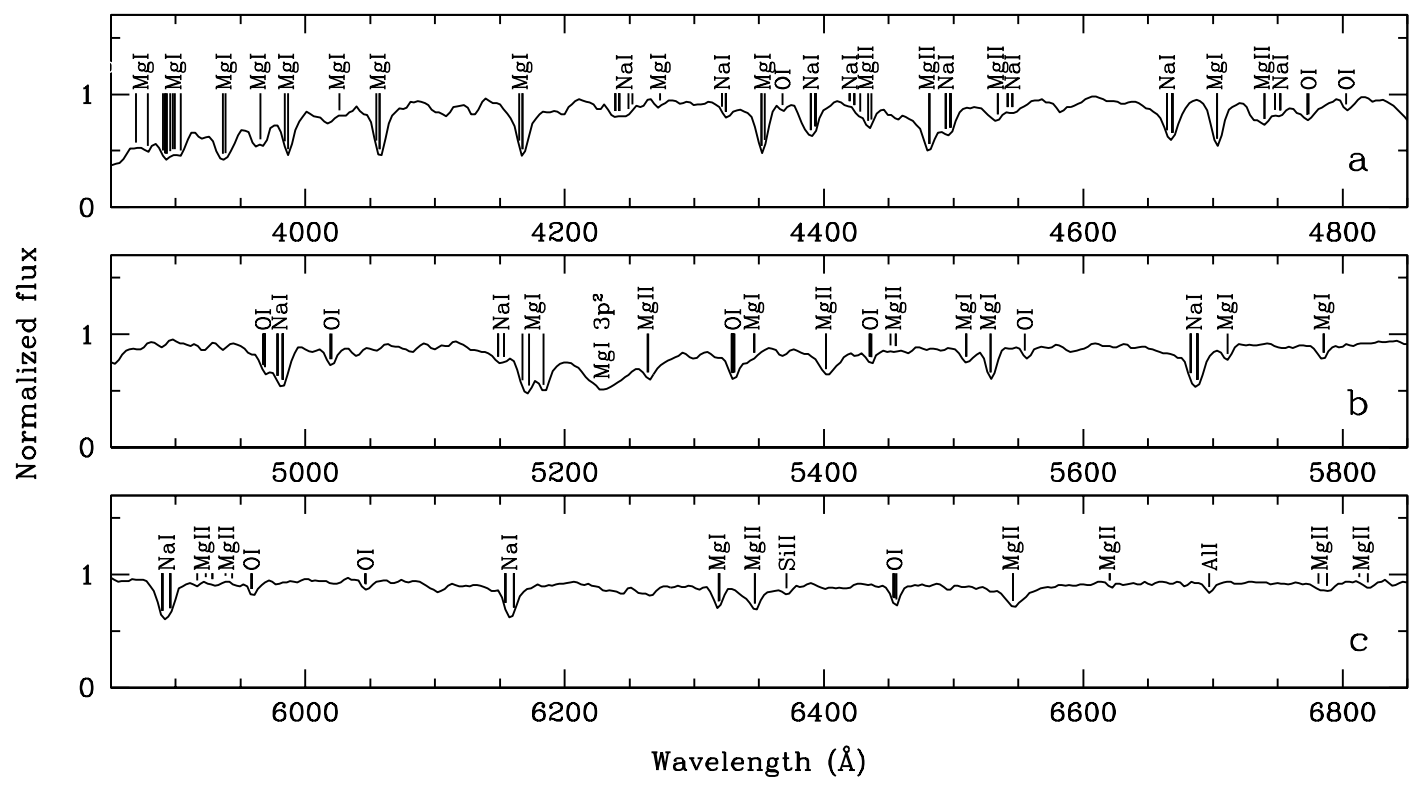

Figure 1: Normalized optical spectrum of LP 40-365 as a function of wavelength. The spectrum was obtained with the Richey-Chretien Spectrograph at the 4-m telescope (KPNO). Dominant spectral lines of sodium ( $\mathrm{Na} \mathrm{I}$ ) and magnesium (Mg I and $\mathrm{Mg}$ II) are labelled along with weaker lines of oxygen (O I), aluminium (Al I) and silicon (Si II). A broad feature near $5230 \AA$ is tentatively identified with a resonance in the $\mathrm{Mg} \mathrm{I} 3 \mathrm{p}^{2}$ photoionization cross section. 


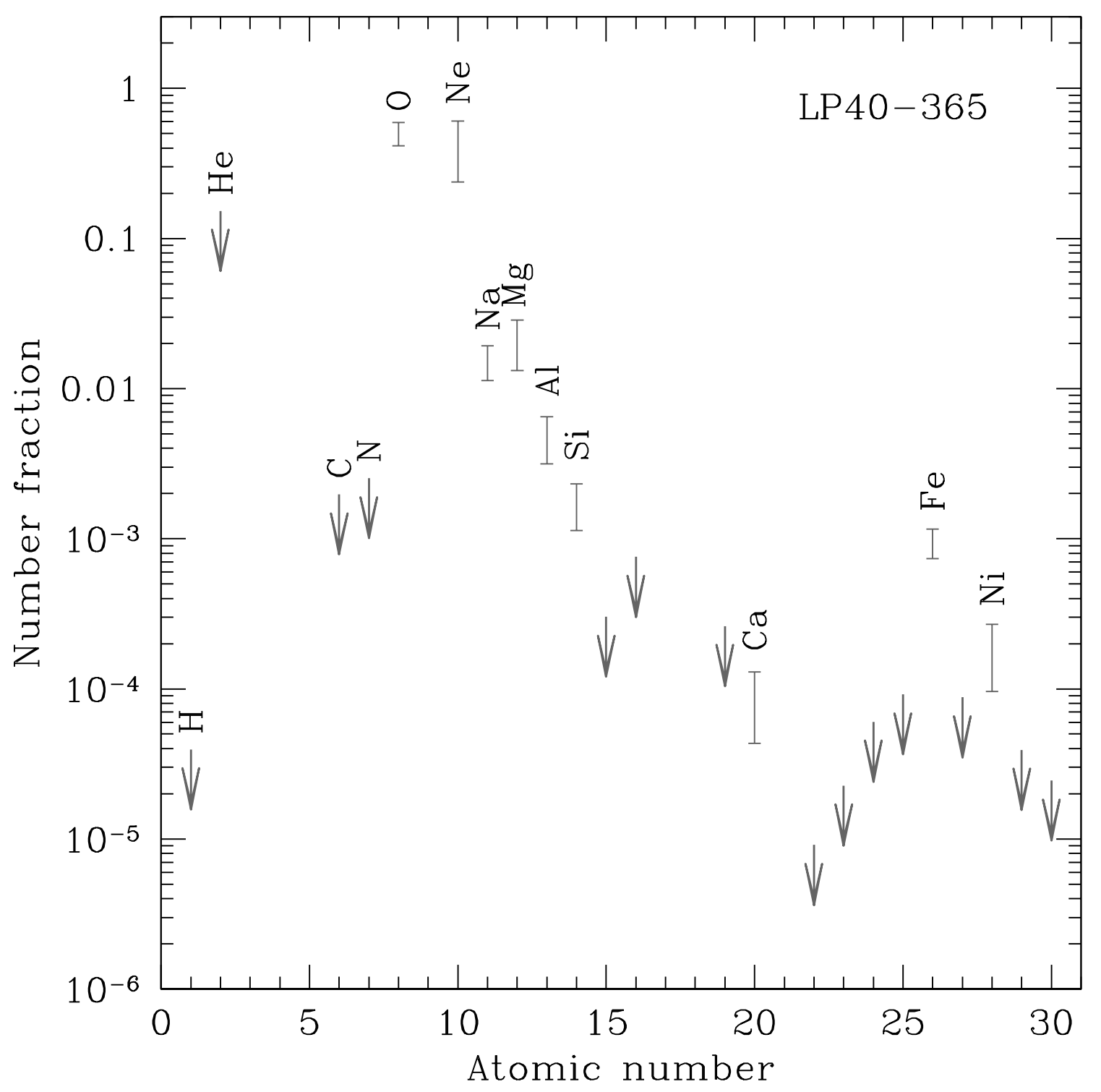

Figure 2: Elemental abundances of LP 40-365. The photospheric abundances, expressed as the number fraction versus the atomic number, were measured in the high-dispersion spectrum obtained with GRACES at the Gemini-North telescope on Mauna Kea. The atmosphere is dominated by oxygen and neon followed by sodium and magnesium. Iron dominates over nickel and other elements in the iron group by at least a factor of 10 . Upper limits are shown with arrows. 

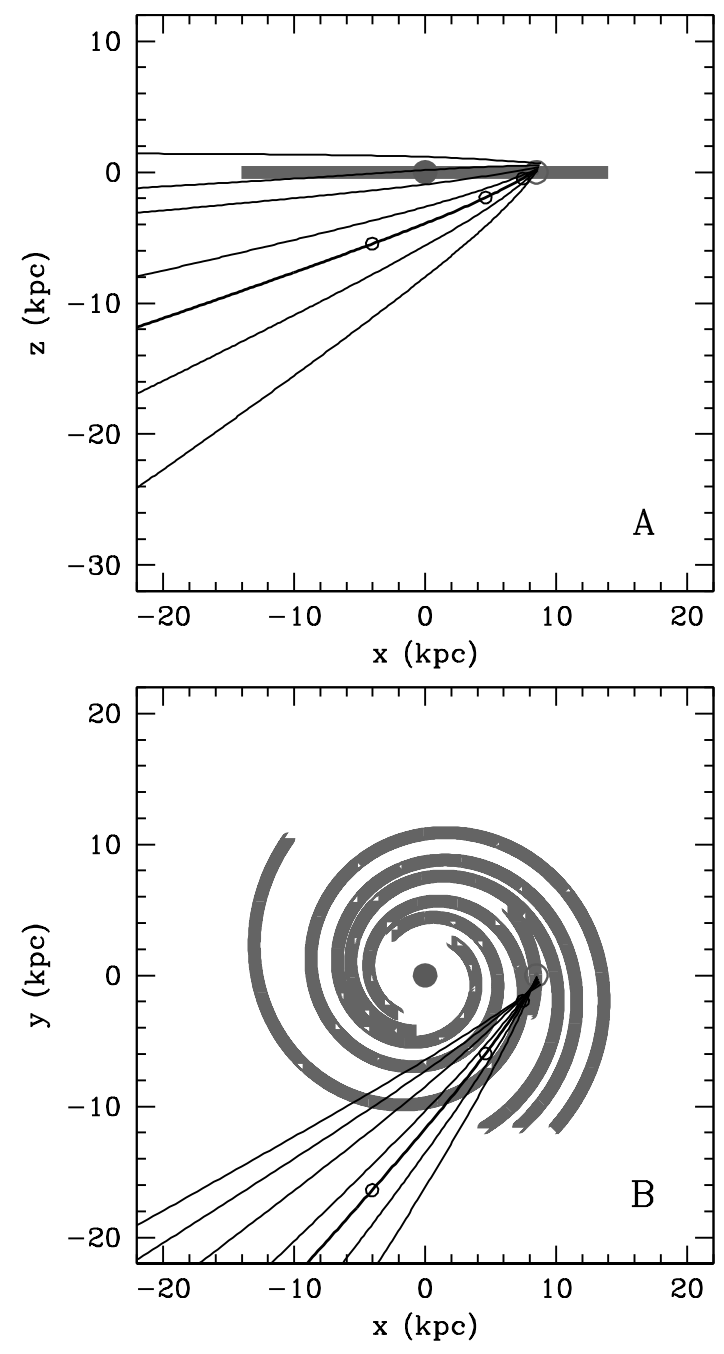

Figure 3: Calculated Galactic motion of LP 40-365. The orbits are drawn (A) in the Galactic plane ( $z$ versus $x$ ) and (B) perpendicular to the plane ( $y$ versus $x$ ) with the GC (solid circle) at the origin. The Sun $(\odot)$ is located $8.5 \mathrm{kpc}$ along the $x$ axis. The current $(t=0)$ position of LP 40-365 is estimated assuming a distance to the Sun of, from uppermost to lowermost curve, $1000,800,600,400,300,200$, and $100 \mathrm{pc}$. The past trajectory resulting from an assumed distance of $300 \mathrm{pc}$ is marked with open circles at, from rightmost to leftmost circle, $-3,-10$ and -30 million years. Schematic views of the Galactic arms are shown in gray. 


\title{
Supplementary Materials for
}

\section{An unusual white dwarf star may be a surviving remnant of a subluminous Type Ia supernova}

\author{
S. Vennes, P. Nemeth, A. Kawka, J.R. Thorstensen, V. Khalack, L. Ferrario, \\ E.H. Alper
}

\section{Supporting Online Material}

\section{Materials and Methods}

S1: Spectroscopic observations and radial velocity measurements

In the course of an on-going spectroscopic survey of sub-luminous, high proper motion stars $(37,38)$, we uncovered a most peculiar object characterized by stellar parameters that would place it below normal main-sequence late-B stars but with a spectrum devoid of hydrogen and helium.

We first observed LP 40-365 on universal time (UT) 2015 February 21 using the RitcheyChretien Focus Spectrograph (RC-spec) (39) attached to the 4-m telescope at Kitt Peak National Observatory (KPNO). We employed the T2KA charge-coupled device (CCD) and the KPC10A grating (316 lines $\mathrm{mm}^{-1}$ ) with a dispersion of $2.75 \AA$ pixel $^{-1}$ in first order and centered on $5300 \AA$. We also inserted the order-sorting filter WG360 and covered a useful spectral range from 3600 to $7200 \AA$. The slit width was set at 1.5 arcsecond resulting in a spectral resolution of $\approx 5.5 \AA$. We obtained one exposure of $1800 \mathrm{~s}$ immediately followed by a second exposure of $1200 \mathrm{~s}$. The observations were conducted near meridian at an airmass $\approx 1.37$ with the slit oriented East-West (position angle $=90^{\circ}$ ) and away from parallactic angle $\left(=160^{\circ}\right)$. The resulting differential slit-loss due to atmospheric dispersion adversely affected the reliability of the flux calibration in the blue part of the spectrum. 
Next, we observed LP 40-365 on UT 2015 June 15-16 with the Intermediate dispersion Spectrograph and Imaging System (ISIS) (40) mounted on the 4.2-m William Herschel Telescope (WHT). We employed the standard dichroic $(5300 \AA)$ to separate and direct the light into the blue and red arms. We used the 1200B and 1200R gratings in the blue and red arms, respectively, and set the slit width to 1 arcsecond providing spectral resolutions of $R=5300$ and $R=9300$, respectively. We obtained five exposures of $1200 \mathrm{~s}$ each spread over two nights to search for variability.

We obtained a set of six spectra from UT 2015 June 23 to July 2 and four additional spectra on UT 2016 Jan 16, using the 2.4-m Hiltner telescope and modular spectrograph (Modspec) (41) at the MDM Observatory on Kitt Peak. We also obtained two additional spectra on UT 2016 Mar 15 using Modspec attached to the MDM 1.3-m McGraw-Hill telescope. A $2048^{2}$ SITe CCD gave $2 \AA$ pixel $^{-1}$ and, with the slit width set at 1.1 arcsecond at the $2.4-\mathrm{m}$ or 1.8 arcsecond at the $1.3-\mathrm{m}$ telescope, provided a $3.5 \AA$ resolution from 4225 to $7560 \AA$.

Finally, LP 40-365 was observed with the Echelle SpectroPolarimetric Device for the Observation of Stars (ESPaDOnS) (42) in the fast-track service mode offered by the Gemini observatory on 2016 June 1 and 4 using the Gemini Remote Access to CFHT ESPaDOnS Spectrograph (GRACES) (43). We adopted the 2-fiber mode which allows for sky-subtraction with a projected width on the sky of 1.2 arcsecond for each fiber. This mode provides a dispersion of 2.88 pixels per resolution element resulting in a resolving power $R=\lambda / \Delta \lambda \approx 40000$ in the 2-fiber mode. The spectrograph nominally covers a range of $6800 \AA$ between 3700 and $10500 \AA$, but we obtained useful data with a signal-to-noise ratio exceeding 40 for 21 orders covering $3750 \AA$ between 4750 and $8500 \AA$. We obtained two exposures of $1955 \mathrm{~s}$ each at an airmass of $\approx 1.75$.

Table S1 lists individual velocity measurements. To obtain the measurements in the lowto intermediate-dispersion data we used the RVIDLINES routine in the RV package within IRAF (44). We employed a set of 15 strong Mg I, Mg II, and Na I spectral lines and applied the method 
to 19 individual spectra obtained with the RC-spec, ISIS/blue and Modspec spectrographs. All 15 spectral lines from the set were employed to measure the velocities with RC-spec, while only 10 and 7 suitable lines were employed with ISIS/blue and Modspec, respectively. Most spectral lines are well separated in the high-dispersion spectra obtained with GRACES. We cross-correlated the spectra with the best-fitting spectral synthesis using the IRAF routine FXCOR. The weighted average and error of the barycentric-corrected radial velocity measurements are given by:

$$
\begin{aligned}
& \bar{v}_{\mathrm{r}}=\frac{\sum_{i=1}^{N} w_{i} v_{\mathrm{r}, i}}{\sum_{i=1}^{N} w_{i}}=497.6 \mathrm{~km} \mathrm{~s}^{-1}, \\
& \sigma_{v_{\mathrm{r}}}=\left(\sum_{i=1}^{N} w_{i}\right)^{-1 / 2}=1.1 \mathrm{~km} \mathrm{~s}^{-1},
\end{aligned}
$$

where $w_{i}=1 / \sigma_{v, i}^{2}$ and $N=21$. The reduced $\chi^{2}$,

$$
\chi_{\mathrm{r}}^{2}=\frac{1}{N-1} \sum_{i=1}^{N} w_{i}\left(v_{\mathrm{r}, i}-\bar{v}_{\mathrm{r}}\right)^{2}=1.3,
$$

indicates that the radial velocity does not vary significantly. Including only higher precision measurements (WHT and Gemini) increases the reduced $\chi_{\mathrm{r}}^{2}$ to $\approx 2.0$ which reveals the effect of small systematic errors of $\approx 3 \mathrm{~km} \mathrm{~s}^{-1}$ in addition to small statistical errors.

\section{S2: Spectral atlas}

Figs. S1, S2, S3, S4, S5, and S6 show details of high-dispersion spectra obtained with GRACES. The echelle spectral orders numbered from 34 to 46 cover a range of $1900 \AA$ between $\lambda=4850$ to $6750 \AA$ and are compared to the best-fitting spectral synthesis. A broad feature near $5230 \AA$ (Fig. 1) is tentatively identified with a resonance in the Mg I $3 \mathrm{p}^{2}$ photoionization cross-section. The available theoretical cross-section included in our spectral synthesis has insufficient wavelength coverage and does not model the observed feature satisfactorily. 
The spectral synthesis includes $\mathrm{H} \alpha$ and $\mathrm{He} \mathrm{I} \lambda 5875$ with hydrogen and helium at their respective abundance upper limit. Numerous lines of Fe I are identified over the entire spectrum, particularly in the blue $(\approx 5000 \AA)$. A few weak spectral lines among many detected in the GRACES spectra remain unidentified or are poorly reproduced by the spectral line synthesis due to inaccurate atomic data or unidentified line blends.

\section{S3: Model atmosphere calculations, fitting techniques, and stellar parameters}

A grid based spectral modelling using a pre-calculated spectral library proved very inefficient due to the peculiar composition of LP 40-365 which would require a prohibitively large number of models to be computed. Therefore we conducted our analysis with the iterative steepest-descent spectral analysis package XTGRID (46) featuring TLUSTY (version 204) (18) and SYNSPEC (version 49) (47), to calculate tailor made model atmospheres and their corresponding synthetic spectra. The atmosphere structure models and spectral synthesis have been performed consistently including the first 30 elements of the periodic table. Helium and the astrophysically important light metals, alpha- and iron-group elements $(\mathrm{C}, \mathrm{N}, \mathrm{O}, \mathrm{Ne}, \mathrm{Na}, \mathrm{Mg}, \mathrm{Al}$, $\mathrm{Si}, \mathrm{S}, \mathrm{Ca}, \mathrm{Fe}, \mathrm{Ni}$ ) were considered in full-NLTE with detailed model atoms, while the rest of the first 30 elements were included assuming LTE conditions. All elements contribute to the electron density, and hence the pressure. Due to the low temperature and high metallicity the stellar plasma is dominated by electrons with contributions from weakly ionized metals, therefore collisions occur predominantly with electrons producing the Stark broadening. Model atoms for low excited ions were taken from the TLUSTY web page (45) and complemented with additional neutral and singly-ionized model atoms (48) and detailed $\mathrm{Mg}$ III and $\mathrm{Na}$ II model atoms (49). We investigated the role of convective energy transport with the TLUSTY models and found that convection is inefficient throughout the atmosphere. 
The XTGRID procedure was initiated with a metal-rich generic extremely low mass WD model, and, by successive approximations along the steepest gradient of the global $\chi^{2}$ surface, it converged on a solution. Each model parameter, such as $T_{\text {eff }}, \log g$, individual abundances, and the projected rotational velocity has been treated separately. To avoid trapping in local minima the procedure regularly increased the step sizes and re-started its descent. The fitting procedure automatically adjusts the atomic data input to the actual model. By evaluating the ionization fractions it assigns the most detailed model atoms to the most populated ionization stages. The spectral analysis was based on the relative line strengths and line profiles. We applied a piecewise normalization of the synthetic spectra together with a linear correction of the flux to match the theoretical continuum to the observed one. The lengths of these fitting ranges were carefully selected to be short around narrow lines and long enough to include the full profiles of strong lines. This approach also reduces the systematics caused by interstellar reddening. We found that the neutral and singly ionized ions, in particular Mg I, Mg II, Fe I, Fe II, Ca I, and $\mathrm{Ca}$ II are the most sensitive temperature indicators, while the surface gravity is constrained by the Stark broadening of the strongest lines. For the spectral synthesis we use the line list available on the SYNSPEC web page and complemented it with the latest atomic transitions data compiled by Robert L. Kurucz (50), and complemented with oscillator strengths of $20 \%$ accuracy or better from the Atomic Spectra Database at the National Institute of Standards and Technology (NIST) (51).

The uncertainties were estimated from the $\chi^{2}$ surface statistics. Models were calculated in one dimension until the reduced $\chi^{2}$ values reached the confidence limit for the given number of free parameters. Parameter correlations are not considered in the error analysis; the procedure exploits these during the iterative fitting, such that parameter correlations in the proximity of the solution are low. The number of independent variables $(p=15)$ in the global fitting procedure accounts for the main stellar parameters $\left(T_{\text {eff }}, \log g\right)$, abundant or spectroscopically 
dominant elements ( $\mathrm{He}, \mathrm{C}, \mathrm{N}, \mathrm{O}, \mathrm{Ne}, \mathrm{Na}, \mathrm{Mg}, \mathrm{Si}, \mathrm{Al}, \mathrm{Ca}, \mathrm{Fe}, \mathrm{Ni}$ ), and the projected rotation velocity $\left(v_{\text {rot }} \sin i\right)$. Error bars are estimated with the condition $\chi_{\alpha}^{2}=\chi_{\min }^{2}+\chi_{p}^{2}(\alpha)$, where $\alpha$ is the significance. Adopting 90\% confidence level, i.e., $\alpha=0.1$ and $\chi_{15}^{2}(0.1)=22.3$, we calculated such errors for the $T_{\text {eff }}, \log g$, and $v_{\text {rot }} \sin i$. We redetermined the individual abundance errors with $90 \%$ confidence using the best-fitting model and keeping all other parameters fixed ( $p=1$, $\left.\chi_{1}^{2}(0.1)=2.7\right)$ and adding in quadrature the measured variations for each abundance during the global fitting procedure.

Our analysis describes the surface properties of LP 40-365, but we have few constraints on its internal structure. We rule out hydrogen and helium because any amount of these elements would quickly diffuse up and contaminate the photospheric layers. The dominant photospheric components, oxygen and neon, are likely core material. Chemical separation would also leave lighter elements such as oxygen and neon on top of heavier elements such as silicon and iron. Fig. S7 shows calculated stellar parameters based on mass-radius relations adopted to estimate the cooling age and mass of the remnant (23). Theoretical radii calculated assuming interior compositions dominated by light elements $(\mathrm{He}, \mathrm{O}, \mathrm{Si})$ but without hydrogen envelopes vary by only a few percent above $0.3 \mathrm{M}_{\odot}(25)$ and the predicted radius inflation is driven by finite temperature effects. A similar behavior is expected at lower mass although it requires extrapolation. Models with an iron core and similarly low-mass are not available but are expected to have, for a given mass, a smaller radius than their light-element counterpart. However, the iron core fraction is unknown. Surface diffusion time scales are comparable to the cooling age $\left(\approx 10^{7} \mathrm{yr}\right)$ of LP 40-365 (29) and without evolutionary computations including the effect of diffusion it is not possible to determine, for example (7), the fraction of the $0.02 \mathrm{M}_{\odot}$ of iron-group material that could have diffused at the center of the $0.28 \mathrm{M}_{\odot} \mathrm{CO}$-dominated core.

By interpolating the cooling tracks (23) depicted in Fig. S7 we converted the surface gravity and temperature measurements into an age of $\approx 5-50 \mathrm{Myr}$ and a mass of $0.13-0.15 \mathrm{M}_{\odot}$. The 
radius and corresponding error bars were estimated following $R=\sqrt{G M / g}$ and the radius error was estimated by allowing $g$ and $M$ to vary within their quoted errors. Error bars for the absolute magnitude were similarly propagated.

\section{S4: Spectral energy distribution}

We obtained ultraviolet photometric measurements $\left(F_{\mathrm{UV}}\right.$ and $\left.N_{\mathrm{UV}}\right)$ from the Galaxy Evolution Explorer (GALEX) all-sky survey (52). We also obtained IR photometric measurements from the Two Micron All Sky Survey (2MASS) (53) and Wide-field Infrared Survey Explorer (WISE) (54). We also include an $R$-band measurement from the Palomar Transient Factory (PTF) photometric catalog 1.0 converted to the Johnson- $R$ photometric band using $R \approx R_{\mathrm{PTF}}-0.14 \mathrm{mag}(55)$. The object was not found to vary with $\sigma\left(R_{\mathrm{PTF}}\right)<0.04$ mag $(56)$. We also include the Sloan $g$ and $r$ and Johnson $V$ and $B$ measurements from the AAVSO Photometric All Sky Survey (APASS) data release number 9 (DR9) (16). Table S2lists the measurements calibrated with either the Vega or AB system as prescribed.

The peak of the spectral energy distribution (Fig. S8) is well reproduced by a spectral synthesis based on parameters measured with the optical spectral line analysis. The concordance validates our conclusions on the properties of this object. Since LP 40-365 lies well outside the Galactic plane, we computed the model spectrum including the effect of the total extinction measured in the line-of-sight, $E_{B-V}=0.027$ (57). The synthetic optical magnitudes $(B, V, R, g, r)=(15.73,15.57,15.51,15.60,15.62)$ mag computed using the model spectrum are

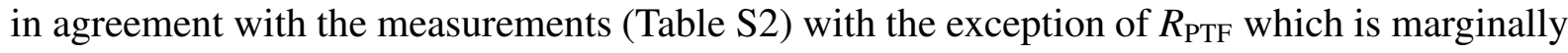
consistent. The synthetic ultraviolet magnitudes $\left(F_{\mathrm{UV}}, N_{\mathrm{UV}}\right)=(21.34,18.01)$ differ from the observed magnitudes although the $F_{\mathrm{UV}}$ measurement is uncertain. The discrepancy in the $N_{\mathrm{UV}}$ magnitudes ( $-0.6 \mathrm{mag})$ is possibly caused by missing ultraviolet opacities in the model calcu- 
lations that would affect the spectral energy distribution.

\section{S5: Stellar kinematics}

For the computation of the orbit of LP 40-365 we have used a code based on the Galactic potential of Allen \& Santillan (27). In a cylindrical coordinate system with origin in the Galactic center this potential is analytical, time-independent and symmetric with respect to the z-axis. This potential consists of three components: a disk and a central bulge of the Miyamoto-Nagai (58) form and a massive spherical halo extending to a radius of $100 \mathrm{kpc}$. The distance of the Sun from the Galactic center is taken to be $8.5 \mathrm{kpc}$. The local circular velocity for this Galactic potential is $V_{0}=220.0 \mathrm{~km} \mathrm{~s}^{-1}$ and the local escape velocity is $V_{\mathrm{esc}}=535.7 \mathrm{~km} \mathrm{~s}^{-1}$.

We have integrated the equations of motion using a fourth order Runge-Kutta algorithm with integration time steps chosen to ensure that the total energy $E_{\text {tot }}$ is conserved with a precision of at least $\Delta E_{\text {tot }} / E<10^{-10}$. The possible orbits of LP 40-365 were then integrated backwards in time over $120 \mathrm{Myr}$, which largely exceeds the longest possible age that we have estimated for LP 40-365.

\section{References and Notes}

37. A. Kawka, S. Vennes, Spectroscopic Identification of Cool White Dwarfs in the Solar Neighborhood. Astrophys. J. 643, 402-415 (2006).

38. A. Kawka, S. Vennes, A study of high proper-motion white dwarfs - I. Spectropolarimetry of a cool hydrogen-rich sample. Mon. Not. R. Astron. Soc. 425, 1394-1412 (2012).

39. A description of the instrument is currently available at https://www.noao.edu/ kpno/manuals/rcspec/rcsp.html. 
40. A description of the instrument is currently available at http://www.ing.iac.es/ astronomy/instruments/isis/.

41. A description of the instrument is currently available at http://mdm.kpno.noao. edu/Manuals/ModSpec/modspec_man.html.

42. N. Manset, J.-F. Donati, ESPaDOnS; an echelle spectro-polarimetric device for the observations of stars, Proc. SPIE 4843, 425-436 (2003).

43. A description of the instrument is currently available at http://www.gemini.edu/ sciops/instruments/graces/.

44. Image Reduction and Analysis Facility (IRAF) is distributed by the National Optical Astronomy Observatories, which are operated by the Association of Universities for Research in Astronomy, Inc., under cooperative agreement with the National Science Foundation.

45. See http://nova.astro.umd.edu/.

46. P. Németh, A. Kawka, S. Vennes, A selection of hot subluminous stars in the GALEX survey - II. Subdwarf atmospheric parameters. Mon. Not. R. Astron. Soc. 427, 2180-2211 (2012).

47. T. Lanz, I. Hubeny, A Grid of NLTE Line-blanketed Model Atmospheres of Early B-Type Stars. Astrophys. J. Supp. Ser. 169, 83-104 (2007).

48. C. Allende Prieto, D. L. Lambert, I. Hubeny, T. Lanz, Non-LTE Model Atmospheres for Late-Type Stars. I. A Collection of Data for Light Neutral and Singly Ionized Atoms. Astrophys. J. Supp. Ser. 147, 363-368 (2003).

49. P. Nemeth, X-ray Modeling of Classical Novae. Bulletin of the American Astronomical Society 42, 371.06 (2010). 
50. See http: //kurucz.harvard.edu/atoms.html.

51. See https://www.nist.gov/pml/atomic-spectra-database.

52. P. Morrissey et al., The Calibration and Data Products of GALEX. Astrophys. J. Supp. Ser. 173, 682-697 (2007).

53. M. F. Skrutskie et al., The Two Micron All Sky Survey (2MASS). Astron. J. 131, 11631183 (2006).

54. E. L. Wright et al., The Wide-field Infrared Survey Explorer (WISE): Mission Description and Initial On-orbit Performance. Astron. J. 140, 1868-1881 (2010).

55. E. O. Ofek et al., The Palomar Transient Factory Photometric Calibration. Publ. Astron. Soc. Pac. 124, 62-73 (2012).

56. E. O. Ofek et al., The Palomar Transient Factory photometric catalog 1.0. Publ. Astron. Soc. Pac. 124, 854-860 (2012).

57. D. J. Schlegel, D. P. Finkbeiner, M. Davis, Maps of Dust Infrared Emission for Use in Estimation of Reddening and Cosmic Microwave Background Radiation Foregrounds. Astrophys. J. 500, 525-553 (1998).

58. M. Miyamoto, R. Nagai, Three-dimensional models for the distribution of mass in galaxies. Pub. Astron. Soc. Japan 27, 533-543 (1975).

\section{Acknowledgments}

Based on observations obtained at the Gemini Observatory, programme GN-2016A-FT-24, using ESPaDOnS, located at the Canada-France-Hawaii Telescope (CFHT). CFHT is operated by the National Research Council of Canada, the Institut National des Sciences de l'Univers of the 
Centre National de la Recherche Scientique of France, and the University of Hawaii. The operations at the Canada-France-Hawaii Telescope are conducted with care and respect from the summit of Maunakea which is a significant cultural and historic site. ESPaDOnS is a collaborative project funded by France (CNRS, MENESR, OMP, LATT), Canada (NSERC), CFHT and ESA. ESPaDOnS was remotely controlled from the Gemini Observatory, which is operated by the Association of Universities for Research in Astronomy, Inc., under a cooperative agreement with the NSF on behalf of the Gemini partnership: the National Science Foundation (United States), the National Research Council (Canada), CONICYT (Chile), Ministerio de Ciencia, Tecnología e Innovación Productiva (Argentina) and Ministério da Ciência, Tecnologia e Inovação (Brazil). Based on observations obtained at Kitt Peak National Observatory, National Optical Astronomy Observatory, which are operated by the Association of Universities for Research in Astronomy under cooperative agreement with the National Science Foundation.

This publication makes use of data products from the Wide-field Infrared Survey Explorer, which is a joint project of the University of California, Los Angeles, and the Jet Propulsion Laboratory/California Institute of Technology, funded by the National Aeronautics and Space Administration. This publication makes use of data products from the Two Micron All Sky Survey, which is a joint project of the University of Massachusetts and the Infrared Processing and Analysis Center/California Institute of Technology, funded by the National Aeronautics and Space Administration and the National Science Foundation. This publication makes use of data products from the AAVSO Photometric All Sky Survey (APASS). Funded by the Robert Martin Ayers Sciences Fund and the National Science Foundation.

The Gemini data are available at https://archive.gemini.edu/searchform/ NotFail/notengineering/cols=CTOWEQ/GN-2016A-FT-24. The KPNO and WHT data may be retrieved at http://archive.noao. edu/search / query (program 2015A0102) and http://casu.ast.cam.ac.uk/casuadc/ingarch/query (program OP- 
TICON 2015A/22), respectively, and specifying the object coordinates. All photometric data may be retrieved at http://vizier.u-strasbg.fr/viz-bin/VizieR using the object coordinates. The MDM data are available at https:// zenodo.org/record/826288 (DOI: 10.5281/zenodo.826288). TLUSTY and SYNSPEC are public codes available at http: // nova.astro. umd.edu/ and the best-fitting spectral synthesis for LP40-365 is available at https://zenodo.org/record/826139 (DOI: 10.5281/zenodo.826139). XTGRID is available through https://astroserver.org/. 

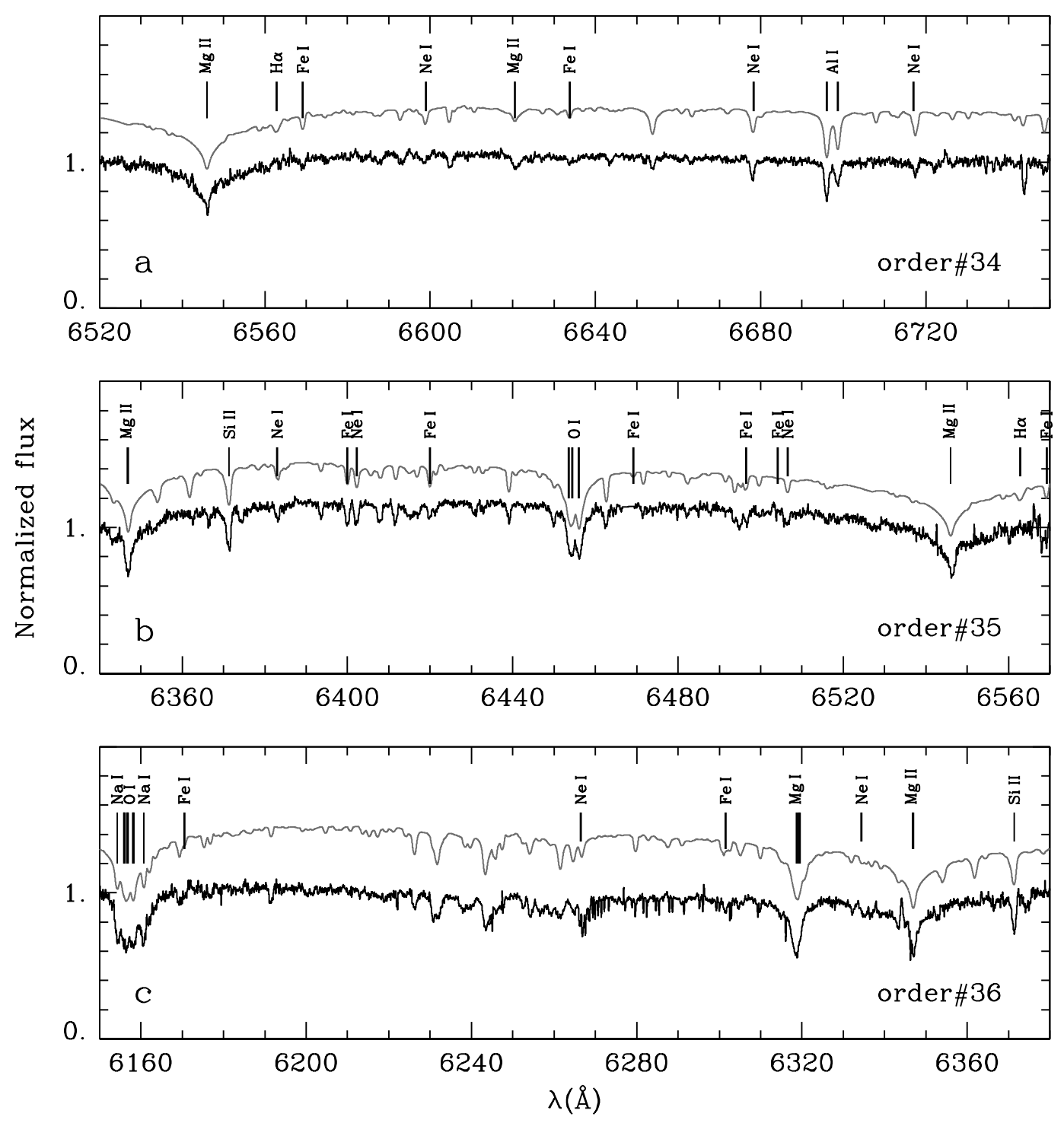

Figure S1: High dispersion spectra and model. Gemini-North high-dispersion spectra covering orders 34 to 36 (lower lines) and normalized to unity. The best-fitting model spectra are shown in grey (top lines) are offset for clarity by +0.3 . Main spectral features are marked. 


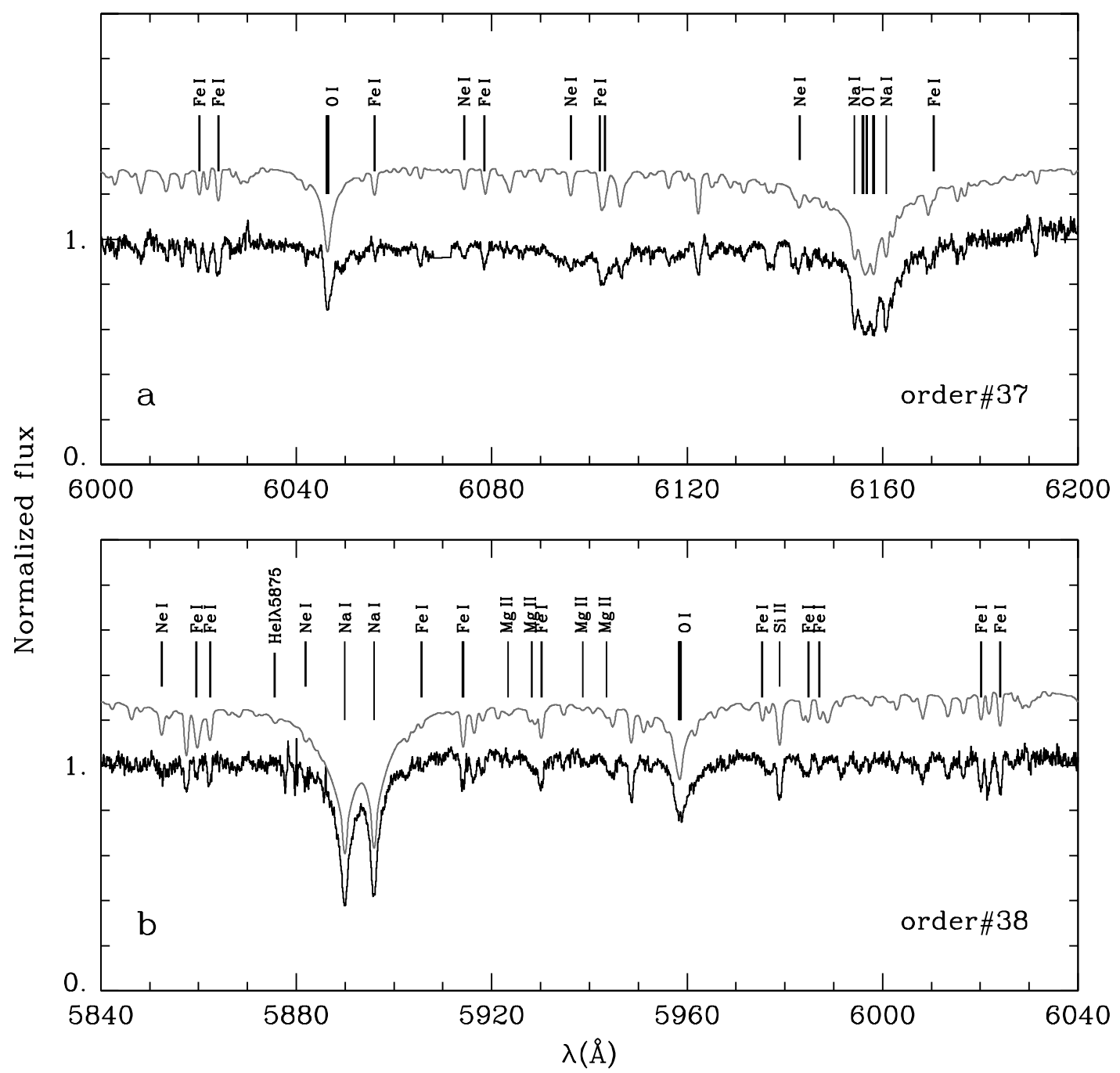

Figure S2: High dispersion spectra and model. Same as Fig. S1 but for orders 37 and 38. 


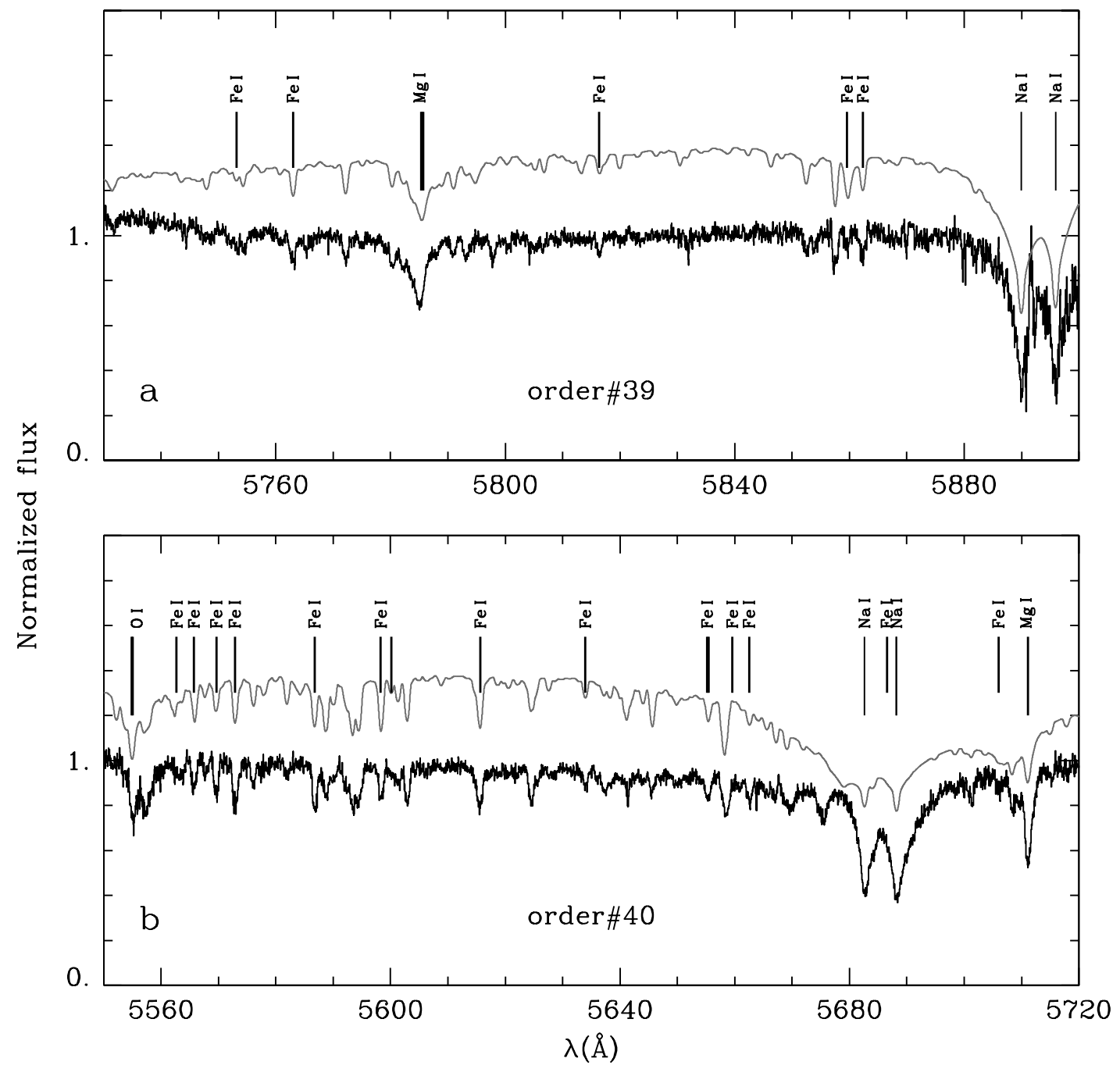

Figure S3: High dispersion spectra and model. Same as Fig. S1 but for orders 39 and 40. 


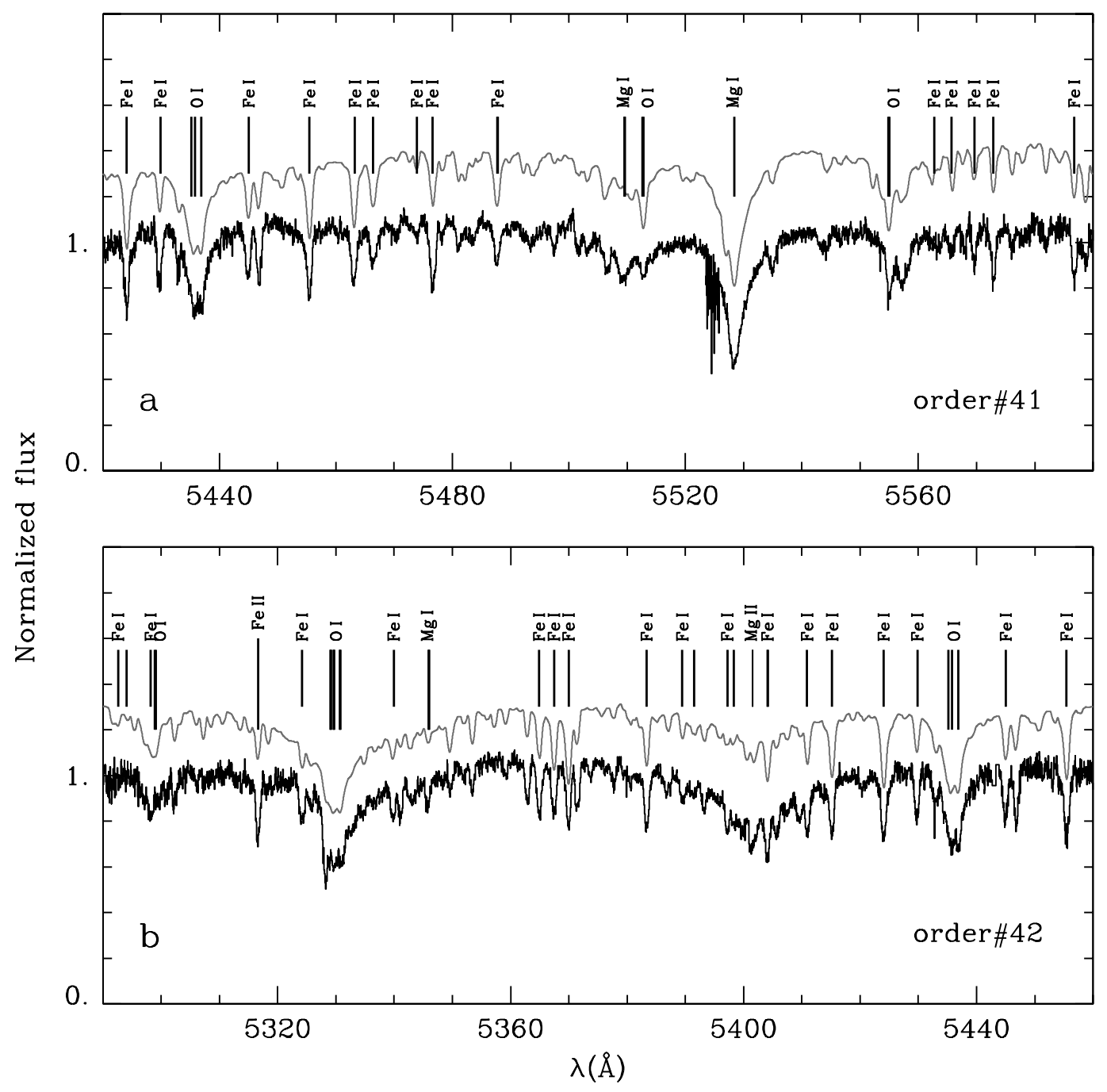

Figure S4: High dispersion spectra and model. Same as Fig. S1 but for orders 41 and 42. 


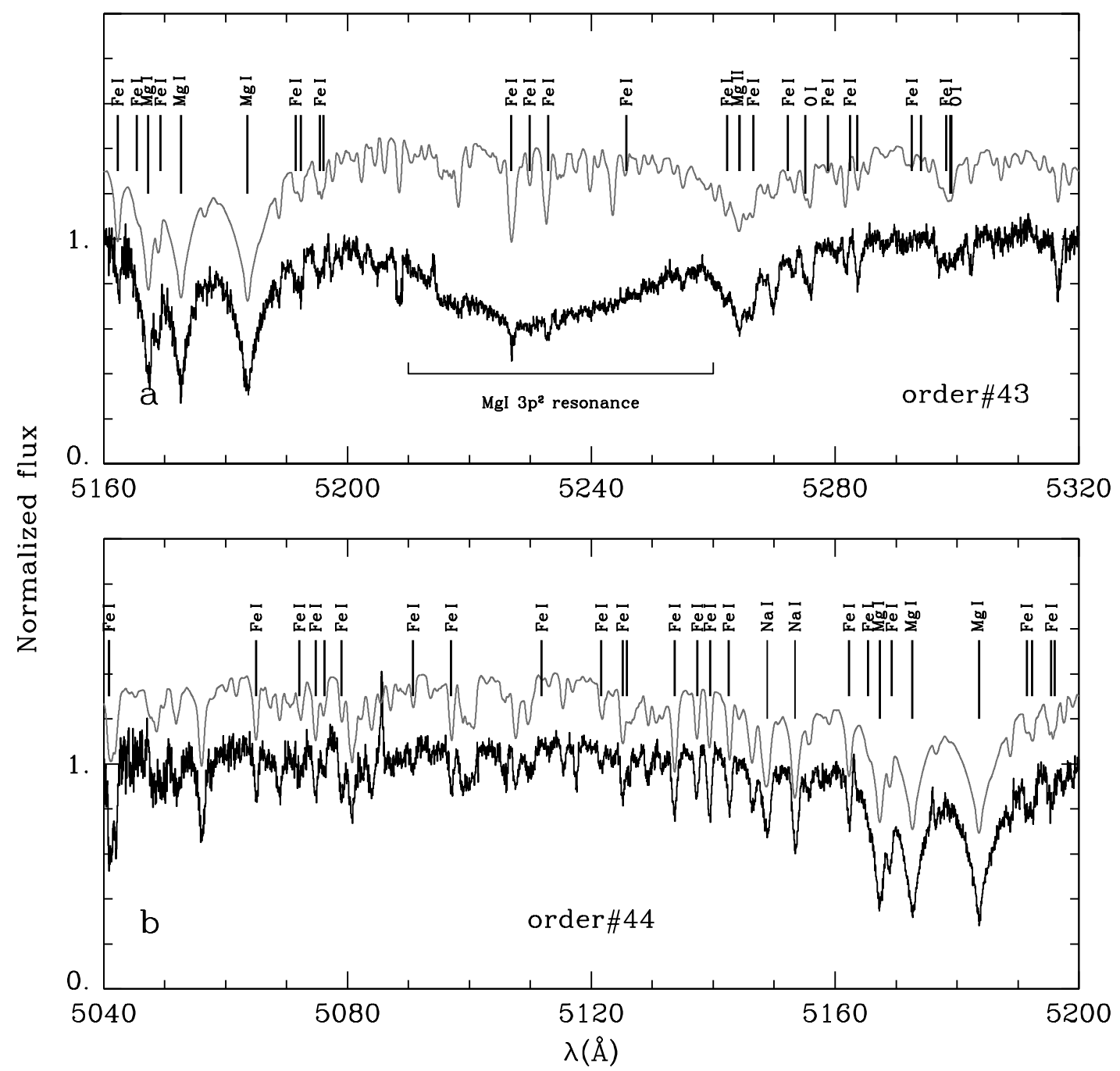

Figure S5: High dispersion spectra and model. Same as Fig. S1 but for orders 43 and 44. 


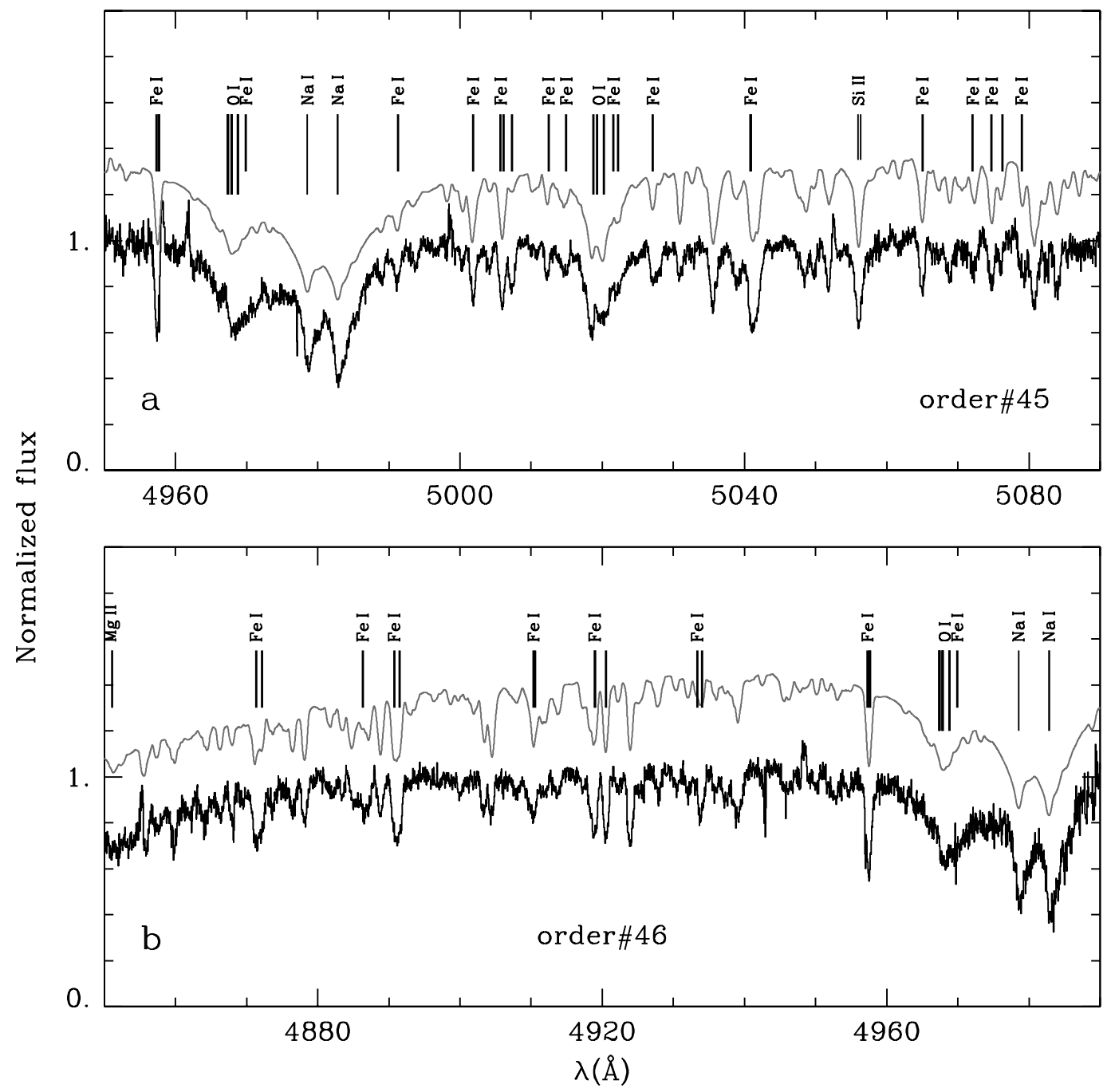

Figure S6: High dispersion spectra and model. Same as Fig. S1 but for orders 45 and 46. 


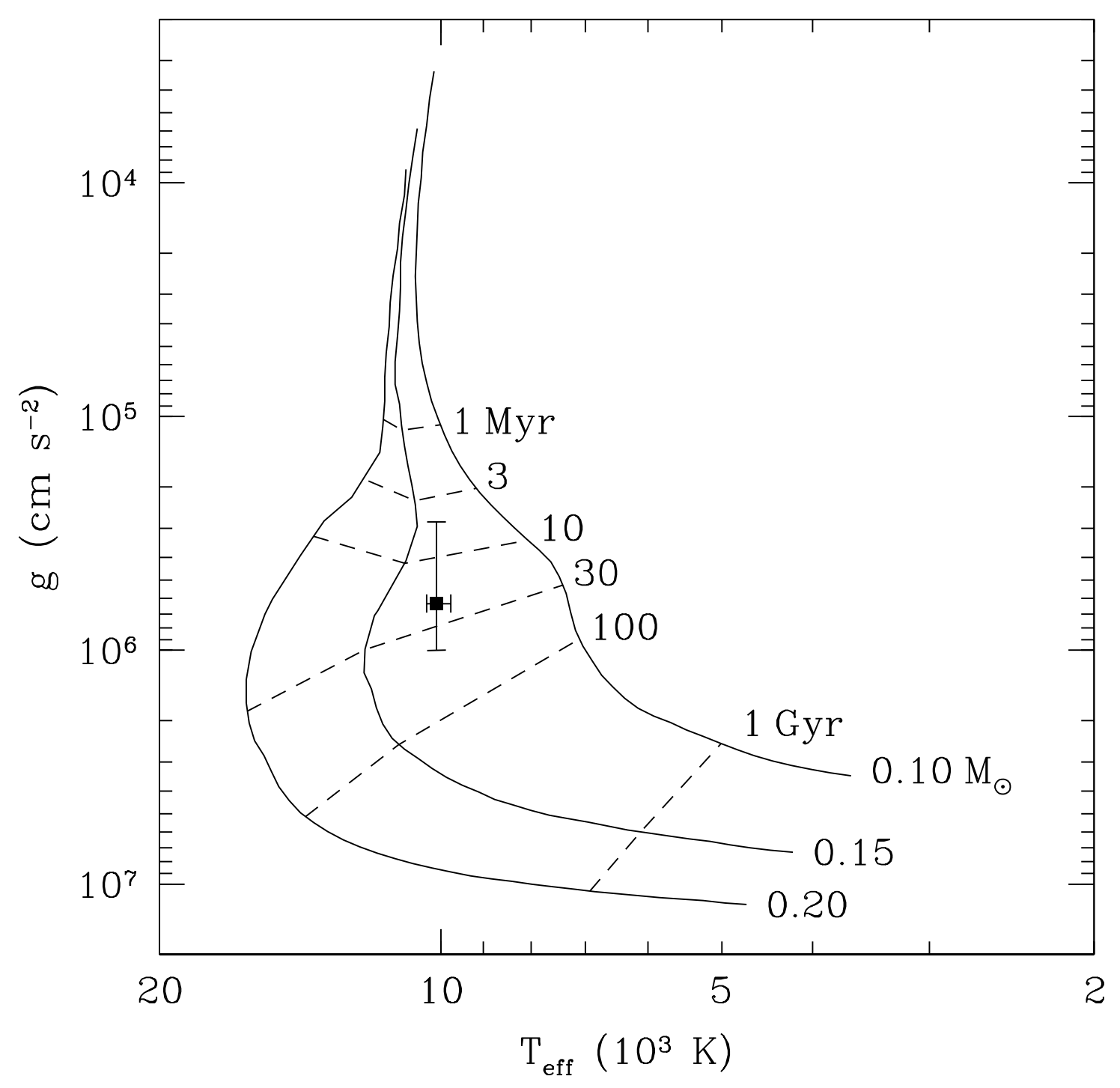

Figure S7: Evolutionary tracks for low mass white dwarfs. The theoretical parameters, surface gravity $(\log g)$ versus effective temperature $\left(T_{\text {eff }}\right)$, for stellar remnants are based on mass-radius relations (23) labelled with the mass (full lines) and cooling ages (dashed lines). The parameters of LP 40-365 are shown with a solid square including error bars. 


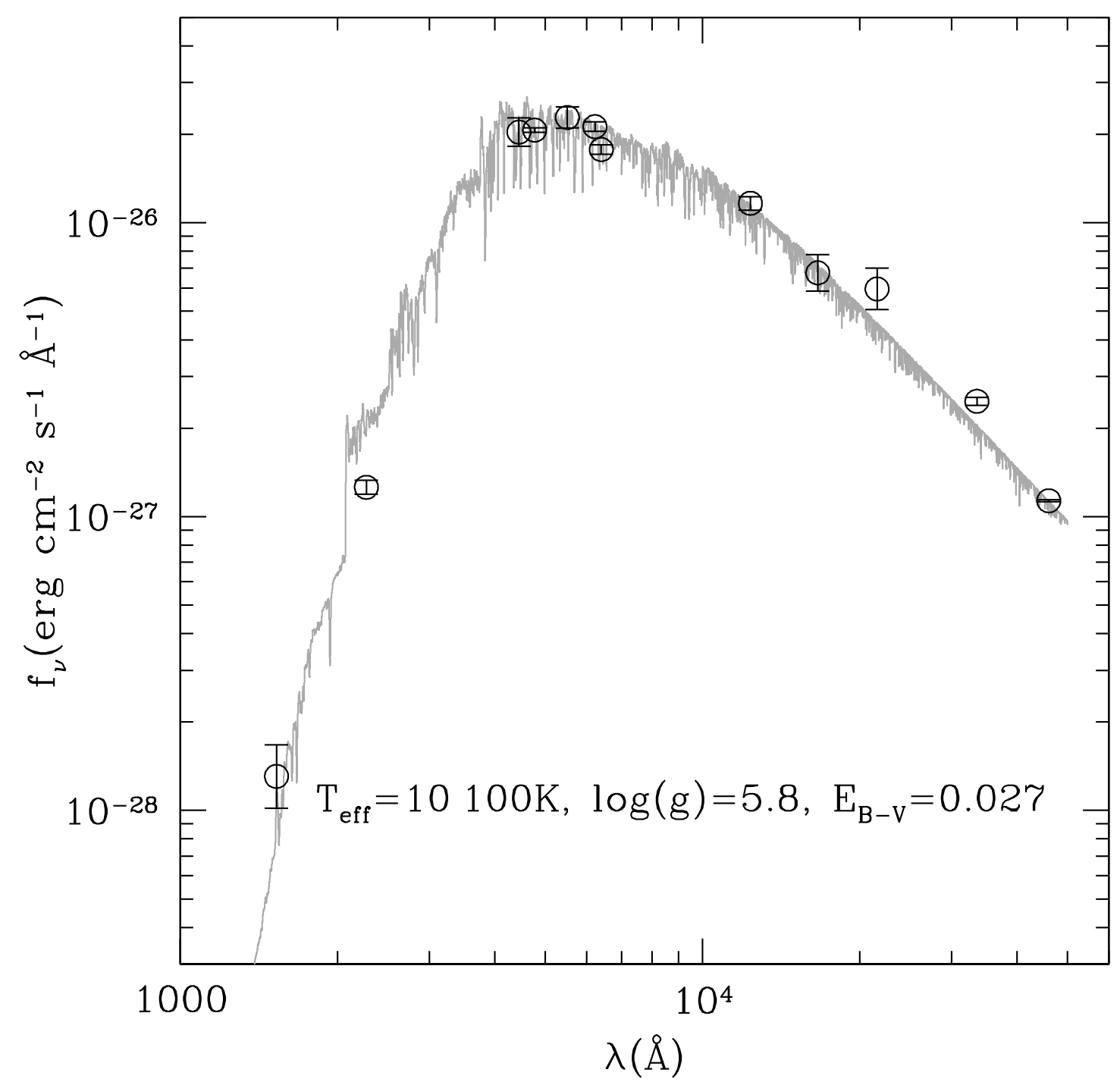

Figure S8: Spectral energy distribution of LP 40-365. The ultraviolet, optical, and infrared photometric measurements (Table (\$2) are shown with open circles and expressed as the flux measured at Earth $\left(f_{v}\right)$ versus wavelength $(\lambda)$ and are compared to a model spectrum (grey line). The adopted stellar parameters $\left(T_{\text {eff }}, \log g\right)$ and the reddening coefficient $\left(E_{B-V}\right)$ applied to the model spectrum are shown. 
Table S1: Barycentric radial velocity measurements. The spectra were obtained at four observatories at the heliocentric julian date (HJD) and were used to measure the radial velocity $\left(v_{\mathrm{r}}\right)$ and the uncertainty on the velocity $\left(\sigma_{v_{\mathrm{r}}}\right)$. Barycentric velocities are corrected to the barycenter of the solar system.

\begin{tabular}{lccr}
\hline Observatory & $\begin{array}{c}\text { HJD } \\
2450000+\end{array}$ & $\begin{array}{c}v_{\mathrm{r}} \\
\left(\mathrm{km} \mathrm{s}^{-1}\right)\end{array}$ & $\begin{array}{r}\sigma_{v_{\mathrm{r}}} \\
\left(\mathrm{km} \mathrm{s}^{-1}\right)\end{array}$ \\
\hline KPNO & 7075.0110 & 515.8 & 11.8 \\
WHT & 7075.0340 & 484.9 & 11.5 \\
& 7189.4283 & 499.0 & 2.0 \\
& 7189.6349 & 505.5 & 4.8 \\
& 7189.6757 & 505.1 & 3.9 \\
MDM & 7190.5045 & 498.8 & 3.4 \\
& 7190.6606 & 502.1 & 3.0 \\
& 7197.6506 & 497.4 & 18.8 \\
& 7197.6593 & 489.1 & 19.4 \\
& 7198.6560 & 470.5 & 27.1 \\
& 7198.6668 & 463.0 & 15.3 \\
& 7205.6543 & 514.0 & 13.8 \\
& 7205.6652 & 515.0 & 24.5 \\
& 7404.0345 & 486.4 & 11.3 \\
& 7404.0453 & 489.0 & 14.3 \\
& 7404.0561 & 485.7 & 17.3 \\
& 7404.0686 & 493.3 & 17.6 \\
Gemini-N & 7462.8685 & 488.4 & 12.0 \\
& 7462.8772 & 499.1 & 10.7 \\
& 7540.7796 & 493.1 & 2.8 \\
\hline
\end{tabular}


Table S2: Photometric measurements. These measurements are used to plot the spectral energy distribution shown in Fig. S8. The conversion into flux units is performed according to the relevant photometric calibration system (AB or Vega).

\begin{tabular}{lcc}
\hline Band & Measurement & System \\
\hline$F_{\mathrm{UV}}$ & $21.11 \pm 0.27 \mathrm{mag}$ & $\mathrm{AB}$ \\
$N_{\mathrm{UV}}$ & $18.65 \pm 0.06 \mathrm{mag}$ & $\mathrm{AB}$ \\
$B$ & $15.80 \pm 0.12 \mathrm{mag}$ & Vega \\
$V$ & $15.51 \pm 0.09 \mathrm{mag}$ & Vega \\
$g$ & $15.61 \pm 0.02 \mathrm{mag}$ & $\mathrm{AB}$ \\
$r$ & $15.58 \pm 0.04 \mathrm{mag}$ & $\mathrm{AB}$ \\
$R$ & $15.60 \pm 0.04 \mathrm{mag}$ & Vega \\
$J$ & $15.34 \pm 0.06 \mathrm{mag}$ & Vega \\
$H$ & $15.47 \pm 0.15 \mathrm{mag}$ & Vega \\
$K$ & $15.12 \pm 0.18 \mathrm{mag}$ & Vega \\
$W 1$ & $15.23 \pm 0.03 \mathrm{mag}$ & Vega \\
$W 2$ & $15.41 \pm 0.07 \mathrm{mag}$ & Vega \\
\hline
\end{tabular}

